\title{
Extreme Risk In Resource Indices And The Generalized Logistic Distribution
}

\author{
Chun-Kai Huang, University of Cape Town \& University of KwaZulu-Natal, South Africa \\ Venelle Pather, University of KwaZulu-Natal, South Africa \\ Jahvaid Hammujuddy, University of KwaZulu-Natal, South Africa \\ Knowledge Chinhamu, University of KwaZulu-Natal, South Africa
}

\begin{abstract}
The resource sector accounts for a substantial proportion of market capitalization on the US and South African stock exchanges. Hence, severe movements in related stock prices can drastically affect the risk profile of the entire market. Extreme value theory provides a basis for evaluating and forecasting such sporadic occurrences. In this article, we compare performances of classical extreme value models against the recently suggested generalized logistic distribution, for estimating value-at-risk and expected shortfall in resource indices. Our results suggest a significant difference in risk behavior between the two markets and the generalized logistic distribution does not always outperform classical models, as previous work may have suggested.
\end{abstract}

Keywords: Extreme Value; Generalized Logistic Distribution; Value-at-Risk; Expected Shortfall; Resource Indices.

\section{INTRODUCTION}

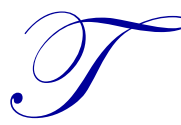

he resource sector plays a major role in both the US and the South African economies. The US has the world's largest coal reserve and is the world's second largest miner of gold and copper. On the other hand, South Africa is estimated to have the world's fifth largest mining sector in terms of GDP value and is also the world's largest supplier of platinum. Both countries are also significant producers of various other natural resources, such as iron, uranium and natural gas. Consequently, the resource sector accounts for a substantial proportion of market capitalization on the countries' respective stock exchanges.

A particular interest is also drawn on the South African resources sector due to recent events. The period 2009-2011 can be characterized as a recovery period from the 2008 global financial crisis. Comparing the relative performance of South Africa to other developing countries, it is safe to say that South Africa has weathered this storm well thus far. This can be attributed to South Africa's low levels of external debt, appropriate fiscal and monetary policies, and a flexible exchange rate. However, unexpected production disruptions caused by labor unrest (for example, the mining strike in 2007, the Marikana strike in 2012, as well as tension in the first half of 2014 due to wage negotiations), skills shortages, safety shutdowns and regulatory uncertainty continue to challenge the South African resource sector. As such, it is interesting to compare extreme risks in the South African resources market to a developed market, such as the US.

An extreme event (i.e., an event that is rare) may have consequences that are either catastrophic or exceptionally rewarding. Examples of such events include earthquakes, tsunamis and winning the national lottery. In finance, extreme events affect aspects such as risk assessment, modeling and management. For example, extreme negative share movements may have a ripple effect on the entire market and increase the likelihood of financial crises. Extreme value theory (EVT) provides the fundamental bases that serve as an appropriate benchmark, encompassing the necessary properties of financial data, as opposed to conventional Gaussian models that often inaccurately describe returns in financial assets. EVT is also advantageous since it focuses on modeling tail behavior. This accommodates for extremal occurrences that may follow an underlying return distribution different from rest of the data.

Koedijk et al. (1990) were the first to utilize EVT in the financial framework and they used it to study the fat-tail behavior in foreign exchange rate returns. More recently, Bali (2003) analyzed the US treasury securities using EVT. 
He investigated the maxima and minima of 3-month, 6-month, 1-year and 10-year US treasury yields and concluded that the fat-tailed Fréchet and Pareto distributions are strongly favored. Embrechts et al. (1999), Brodin and Kluppelberg (2008) and Levine (2009) explored EVT as a risk management tool. Other studies of EVT, in connection with value-at-risk (VaR), are those of Danielsson and de Vries (2000) and Fernandez (2003). From a South African point of view, notable works are those of Seymour and Polakow (2003), Shapiro (2012) and Chinhamu et al. (2015). The performances of EVT in different emerging markets are also investigated by Gençay and Selçuk (2004) and Anđelić et al. (2010).

As an alternative to the generalized extreme value distribution (GEVD), the generalized logistic distribution (GLD) was recently proposed as a limiting distribution of block maxima (BM). Gettinby et al. (2006) studied the share returns of UK, US and Japan stock markets, and concluded that these stock markets were comparable in the sense that their share returns were all appropriately fitted by GLD. Other studies that resulted in favor of the GLD were provided by Tolikas and Brown (2006) for the Athens stock exchange, Tolikas et al. (2007) for the German stock market and Tolikas and Gettinby (2009) for the Singapore stock market. Popularity of the GLD stems from the fact that it has fat tails that are comparable to those of the GEVD and the generalized Pareto distribution (GPD) that arises from the peaks-over-threshold (POT) method. Theoretical aspects of the GLD, as an EVT model for BM, were discussed by Nidhin and Chandran (2013).

The contribution of this article is two-fold. Firstly, we apply the aforementioned EVT models to capture market risk in resource indices for both US and South Africa, where such analysis is scarce, and this allows a sample comparison of the model performances between a developed market and an emerging market. Secondly, evaluations of GLD against GPD performances are drawn. It has been suggested that GLD and GEVD outperforms GPD, for financial risk modeling, by some of the work referenced above. However, our empirical analyses here indicate otherwise. The model performances are compared via their ability in estimating financial risk measures. Specifically, we estimate VaR and expected shortfall (ES), as per international Basel regulatory framework, using these EVT models. The data used are Dow Jones US Mining Index, South African (SA) Mining Index (J177) and JSE Resource 10 Index (J210, RESI).

The remainder of the article is structured as follows. Section II provides the methodological framework for EVT. VaR and ES, together with their respective backtesting procedures, are introduced in Section III. Section IV presents our descriptive and statistical analyses, with detailed discussions on model performances. Lastly, Section $\mathrm{V}$ concludes the article and gives some suggestions for further work.

\section{EXTREME VALUE MODELS}

In this section, we present the two classical ways of modeling extreme data: the BM method and the POT method. Extremes in a data series are characterized by the limiting distribution of these methods (i.e., the distribution of the sample maximum and the distribution of exceedances above a given threshold, respectively). Consequently, we introduce the three limiting distributions: GEVD, GPD and GLD.

Suppose $X_{i}$ is a sequence of random variables that represents the daily returns of some financial asset. The BM method requires the selection of the maximum value in each successive period of a predetermined block size. These chosen observations, called the block maxima, correspond to the most extreme event per period (see Figure 1(a) for an example). On the other hand, the POT method focuses on observations exceeding a specified large threshold value $u$. For example, consider 20 daily observations as depicted in Figure 1(b). Now assume we choose $u$ to be the $80^{\text {th }}$ percentile and, hence, there are 4 observations above $u$. From the figure, we notice that each of $X_{3}, X_{7}, X_{18}$ and $X_{19}$ exceed the threshold $u$ and thus they are referred to as exceedances. For each of these four observations, we compute the exceedances above $u$ (i.e., $X_{3}-u, X_{7}-u, X_{18}-u, X_{19}-u$ ), which are random observations characterized by a distribution of exceedances. 
Figure 1. An example to illustrate the difference between BM (left) and POT (right) approaches

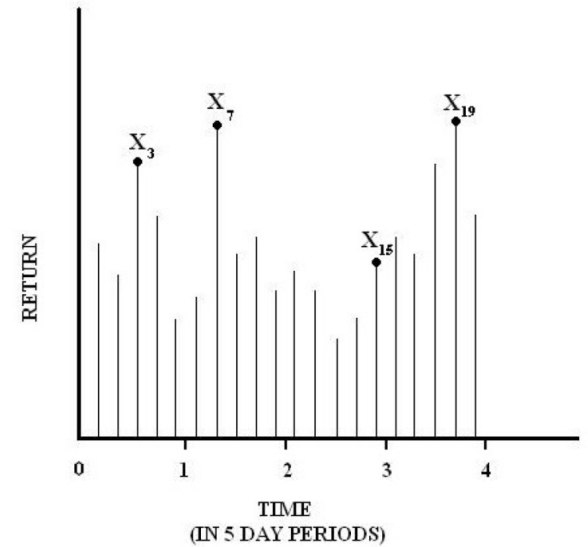

(a)

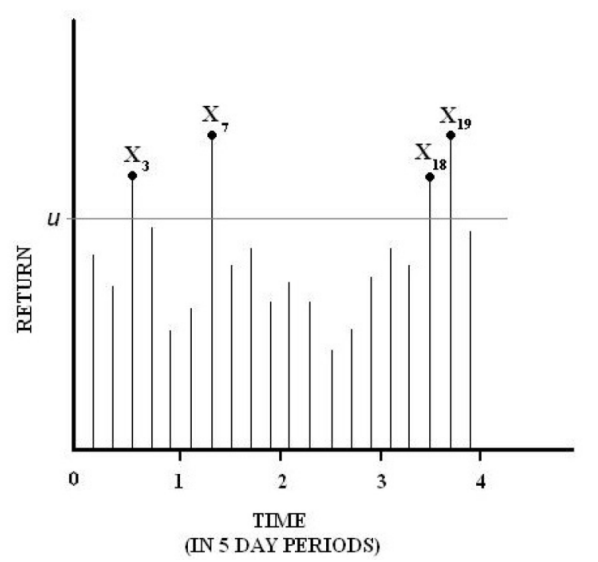

(b)

Formally, let $X_{i}$ be a sequence of independent and identically distributed (i.i.d.) random variables and let $M_{T}$ denote the maximum of $X_{i}, i=1, \ldots, T$. According to Fisher and Tippett (1928) and Gnedenko (1943), regardless of the original distribution of $X_{i}$, the limiting distribution of properly normalized $M_{T}$ is given by GEVD, i.e.,

$$
F_{G E V D}(x)= \begin{cases}\exp \left\{-\left[1+\xi\left(\frac{x-\mu}{\sigma}\right)\right]^{-1 / \xi}\right\} & \text { if } \xi \neq 0 \\ \exp \left[-\exp \left(-\frac{x-\mu}{\sigma}\right)\right] & \text { if } \xi \rightarrow 0\end{cases}
$$

where $\sigma>0$ and $1+\xi\left(\frac{x-\mu}{\sigma}\right)>0 . \mu$ is the location parameter, $\sigma$ is the scale parameter and $\xi$ is the shape parameter. When $\xi>0, F$ belongs to the heavy-tailed Fréchet class of distributions. When $\xi<0, F$ belongs to the short-tailed Weibull class of distributions. And, as $\xi \rightarrow 0, F$ tends to the light-tailed Gumbel class of distributions. Practically, we divide the data into non-overlapping blocks and identify the maximum in each block (i.e., BM). Subsequently, maximum likelihood estimation (MLE) is utilized to find the parameter estimates for $\mu, \sigma$ and $\xi$ (Coles, 2001).

Nidhin and Chandran (2013) has provided the theoretical framework for a generalization to the BM approach. If the sample size $T$ above is assumed to be a random variable following a geometric distribution, independent of $X_{i}$, then the distribution of $M_{T}$ tends to GLD, i.e.,

$$
F_{G L D}(x)= \begin{cases}\left\{1+\left[1-\xi\left(\frac{x-\mu}{\sigma}\right)\right]^{1 / \xi}\right. & \text { if } \xi \neq 0 \\ {\left[1+\exp \left(-\frac{x-\mu}{\sigma}\right)\right]^{-1}} & \text { if } \xi \rightarrow 0\end{cases}
$$

It was shown that GLD is characterized by max-stability and min-stability with respect to the geometric distribution. Furthermore, GLD has tails that are asymptotically equivalent to those of GEVD. The procedure for GLD parameter estimation using MLE is also parallel to that of GEVD.

For the POT method, we assume $X_{i}$ as above and let $u$ be a predetermined high threshold value. Balkema and de Haan (1974) and Pickands (1975) have shown that the conditional probability $P(X-u \leq x \mid X>u)$ can be approximated by GPD, i.e., 


$$
F_{G P D}(x)= \begin{cases}1-\left(1+\frac{\xi x}{\beta}\right)^{-1 / \xi} & \text { if } \xi \neq 0 \\ 1-\exp \left(-\frac{x}{\beta}\right) & \text { if } \xi \rightarrow 0\end{cases}
$$

as $u$ tends to the right end point of $X$. For this distribution, $x>0$ when $\xi \geq 0,0 \leq x \leq-\beta / \xi$ when $\xi<0$, and $\beta>0$. To estimate the parameters, we first identify those values that lie above $u$ and calculate $x-u$, the exceedances. Subsequently, MLE is executed using these exceedances and estimates for $\beta$, and $\xi$, are obtained (Coles, 2001).

\section{RISK MEASURES}

Financial, as well as non-financial, institutions can individually, or collectively, cause an effect which results in a significant impact on the economy as a whole. Consequently, risk is unpredictable and unavoidable. Although, we can prepare for it so that if we succumb to it then at least the fall will be cushioned. A central characteristic can also be traced back to numerous financial disasters; that is, the inadequacy of supervision and lack of risk management can quickly spiral towards thwarted losses. As such, VaR has become a benchmark for many institutions to easily and effectively quantify market risk. However, VaR's inability to capture some aspects of market risk, such as tail loss and subadditivity, has led to the Basel Committee on Banking Supervision recommending a shift of focus to the alternative ES measure. The use of ES has been suggested, in addition to VaR, for the internal model-based approach and to be utilized in determining risk loads for the standardized approach (Basel, 2012).

$\mathrm{VaR}$ is defined as the maximum loss of a portfolio such that the probability of a loss exceeding that amount, over a specified risk horizon, is equal to a pre-specified tolerance level. Formally, suppose $X$ (usually the return in some risky financial instrument) is a random variable with distribution function $F$. VaR over a specified time period, for a given probability $\alpha$, can be defined as the $\alpha$-th quantile of $F$, i.e.,

$$
\operatorname{VaR}_{\alpha}=F^{-1}(\alpha)
$$

where $F^{-1}$ is the corresponding quantile function. As for the EVT approach, we can estimate VaR of the original returns using quantiles of the fitted GEVD, GLD and GPD models.

For GEVD and GLD, we can utilize the fact that block maxima are obtained over non-overlapping periods of a specified length. Hence, we may convert an estimated GEVD, or GLD, quantile to a VaR estimate of the daily returns distribution by adjusting the confidence level $\alpha$. Observe that $\alpha$ is the probability that a daily return will exceed $\operatorname{VaR}_{\alpha}$, i.e., $P\left(X>\operatorname{VaR}_{\alpha}\right)$ and let $\alpha_{\text {ext }}$ be the probability that a block maxima, observed over a period of $T$ days, will exceed $\operatorname{VaR}_{\alpha}$, i.e., $P\left(M_{T}>\operatorname{VaR}_{\alpha}\right)$. Then we have

$$
\alpha_{\text {ext }}=P\left(M_{T}>\operatorname{VaR}\right)=1-P(\text { all } X<\operatorname{VaR})=1-(1-\alpha)^{T}
$$

In other words, if we want to estimate $\mathrm{VaR}$ at level $\alpha$ (for the original daily returns data), we can simply compute the quantile from GEVD, or GLD, at $\alpha_{\text {ext }}$.

For GPD, we first define $F_{u}(x)=P(X-u \leq x \mid X>u)$, where $x$ represents the size of exceedances over a specified threshold $u$. Then, we may deduce the reverse expression

$$
F(x)=(1-F(u)) F_{u}(x-u)+F(u)
$$

Given the choice of a sufficiently high threshold, we can estimate $F(u)$ by $\left(1-N_{u} / n\right)$, where $n$ is the total number of daily returns in our data and $N_{u}$ is the number of observations above the chosen threshold. And, $F_{u}(x-u)$ can be estimated by a GPD using maximum likelihood estimation (Embrechts et al., 1997). Hence, we can estimate the inverse probability $F^{-1}(\alpha)$. 
ES, or conditional $\mathrm{VaR}$, is the mean of returns that exceed a corresponding $\mathrm{VaR}$ value, i.e., for a given confidence level $\alpha, \mathrm{ES}_{\alpha}=E\left(X \mid X>\operatorname{VaR}_{\alpha}\right)$. This expression can be estimated by

$$
\widehat{\mathrm{ES}}_{\alpha}=\frac{\sum_{1}^{n} X_{i} I_{\left[X_{i}>\widehat{\operatorname{VaR}}_{\alpha}\right]}}{\sum_{1}^{n} I_{\left[X_{i}>\widehat{\operatorname{VaR}}_{\alpha}\right]}}
$$

where $\widehat{\operatorname{VaR}}_{\alpha}$ is the estimated $\mathrm{VaR}$ at level $\alpha$ and $I_{\left[X_{i}>\overrightarrow{V a R}_{\alpha}\right]}$ is an indicator variable on the event $\left\{X_{i}>\widehat{\operatorname{VaR}}_{\alpha}\right\}$. As an alternative risk measure to VaR, ES takes into consideration both the likelihood of exceedance above a threshold and the size of the exceedance (Acerbi and Tasche, 2002).

To examine the suitability and effectiveness of VaR and ES estimates derived from our models, we apply backtesting procedures. Specifically, backtesting for VaR is accomplished by using the Kupiec likelihood ratio test (Kupiec, 1995). Whereas, we follow the procedure in McNeil and Frey (2000), with bootstrapping, for ES backtesting.

The Kupiec test uses the fact that a suitable model ought to have its proportion of violations of VaR estimates close to the corresponding tail probability level. The method consists of calculating $x^{\alpha}$, the number of times the observed returns exceed the $\mathrm{VaR}$ estimate at level $\alpha$, i.e., $X_{i}>\widehat{V a R}_{\alpha}$, and compare the corresponding failure rates to $\alpha$. The null hypothesis is that the expected proportion of violations is equal to $\alpha$ and the Kupiec statistic, given by

$$
K=2 \ln \left[\left(\frac{x^{\alpha}}{N}\right)^{x^{\alpha}}\left(1-\frac{x^{\alpha}}{N}\right)^{N-x^{\alpha}}\right]-2 \ln \left[\alpha^{x^{\alpha}}(1-\alpha)^{N-x^{\alpha}}\right]
$$

is asymptotically distributed according to a chi-square distribution with one degree of freedom.

The null hypothesis of the ES backtest is that the excess conditional shortfalls (excess of the actual data series when $\mathrm{VaR}$ is violated), are i.i.d. and has zero mean. The test is a onesided $t$-test against the alternative that the excess shortfall has mean greater than zero and thus that the conditional shortfall is systematically underestimated. The test statistic is given by

$$
T=\frac{\bar{r}-\mu_{0}}{\bar{\sigma} / \sqrt{m}}
$$

where $\bar{r}$ and $\bar{\sigma}$ are the mean and standard deviation of exceedance residuals $\left\{r_{1}, r_{2}, \ldots, r_{m}\right\}$. The bootstrap techniques can also be utilized to alleviate any bias with respect to assumptions about the underlying distribution of the excess shortfall. For the bootstrap test, we sample $\left\{\tilde{r}_{1}^{*}, \tilde{r}_{2}^{*}, \ldots, \tilde{r}_{m}^{*}\right\}$ without replacement from the shifted residuals $\tilde{r}_{i}=r_{i}-$ $\bar{r}+\mu_{0}$ and compute the test statistic

$$
T_{j}^{*}=\frac{\overline{\tilde{r}}^{*}-\mu_{0}}{\overline{\tilde{\sigma}} / \sqrt{m}}
$$

for each bootstrap sample $j$ (McNeil and Frey, 2000).

\section{DATA ANALYSIS}

The data used in our analyses are extracted from McGregor BFA. These include the daily closing prices of US Mining Index (in US Dollars), SA Mining Index (in South African Rands) and RESI (in South African Rands). These data were converted to natural logarithmic returns (or simply 'returns'), which is defined as

$$
V_{i}=\ln \left(T_{i} / T_{i-1}\right)
$$


where $T_{i}$ is the closing index price on day $i$. For our in-sample analyses, the Dow Jones US Mining Index returns date from 25 June 2004 to 24 June 2014 (2608 daily returns) and both South African Indices were recorded from 10 September 2004 to 9 September 2014 (2500 daily returns).

Table 1 below presents the summary statistics of the three indices. It is observed that JSE Resource 10 Index has the highest mean $(0.0414 \%)$, followed by SA Mining Index $(0.0358 \%)$, both of which are only marginally positive. This is an indication that both time series have slightly increased over the period under consideration. It is also observed that the most volatile index is the US Mining Index, with a standard deviation of $2.533 \%$, while that of the SA Mining Index and RESI are quite close to each other, at $1.949 \%$ and $1.8805 \%$ respectively.

Considering skewness, we notice that the SA and US Mining Index returns are both skewed to the right (with US Mining Index having a relatively higher skewness), while the opposite is true for RESI. The excess kurtosis values indicate the leptokurtic behavior of these return series. This means that the empirical distributions of the daily returns have a much fatter tail than that of the Gaussian distribution. This can also be verified by the Jarque-Bera statistic (and corresponding $p$-values) which are high enough (close to zero) to reject the normality assumption at all significance levels.

Table 1. Descriptive statistics for daily returns in Dow Jones US Mining Index (US-MINI), SA Mining Index (JSE-MINI) and JSE Resource 10 Index (JSE-RESI10)

\begin{tabular}{|l|l|l|l|l|l|l|l|l}
\hline \multicolumn{1}{c|}{ Index } & $\mathbf{N}$ & Minimum & Maximum & $\begin{array}{c}\text { Excess } \\
\text { Kurtosis }\end{array}$ & Skewness & Mean & $\begin{array}{c}\text { Jarque-Bera } \\
\text { Statistic }(\boldsymbol{p} \text {-value })\end{array}$ & $\begin{array}{c}\text { Standard } \\
\text { Deviation }\end{array}$ \\
\hline US MINING & 2608 & -0.177527 & 0.171462 & 6.52454 & 0.394862 & -0.00001 & $\begin{array}{l}4704.1203 \\
(<0.0001)\end{array}$ & 0.025330 \\
\hline SA MINING & 2500 & -0.119659 & 0.116163 & 4.39827 & 0.004681 & 0.00036 & $\begin{array}{l}2020.5172 \\
(<0.0001)\end{array}$ & 0.019490 \\
\hline RESI & 2500 & -0.118154 & 0.114998 & 4.62447 & -0.018585 & 0.00041 & $\begin{array}{l}2233.7058 \\
(<0.0001)\end{array}$ & 0.018805 \\
\hline
\end{tabular}

These basic results are properties of financial series that provide a justification to use GEVD, GLD and GPD for modeling the data at hand. Similar properties may be observed in the time series plots of the data, as provided in Figure 2. These also include some observable extremal events, such as effects of the 2008 global financial crisis.

Figure 2. Time series plots for daily returns in (a) Dow Jones US Mining Index, (b) SA Mining Index and (c) JSE Resource 10 Index

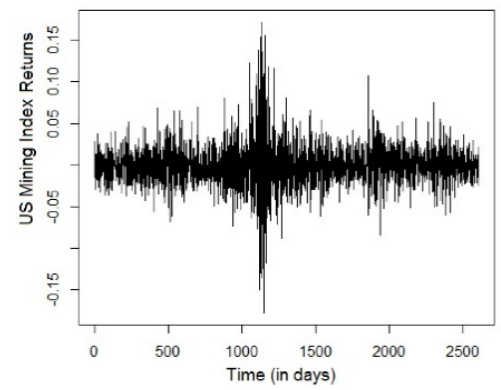

(a)

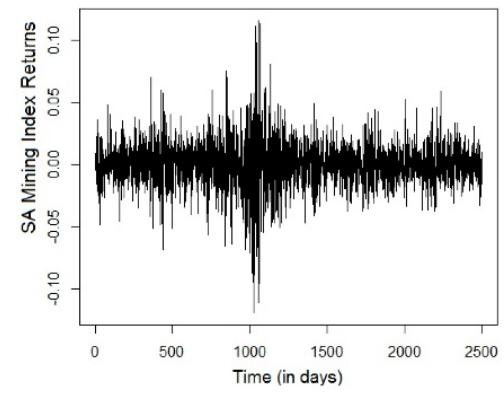

(b)

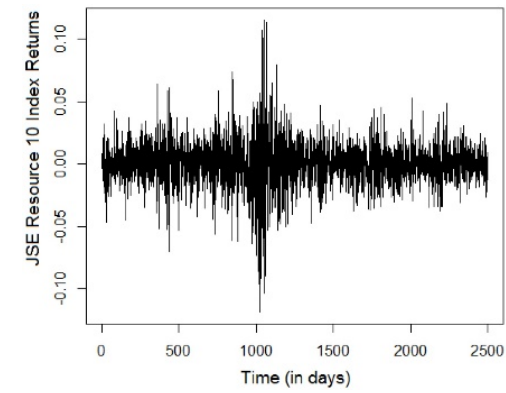

(c)

Large negative returns are the main concern in the field of financial risk management. For example, it may signify the situation of a stock market crash. They are also directly connected to the adequacy of risk capital set aside by financial institutions. Hence, our analyses here focus solely on extreme losses, although the methodologies can easily translate to large positive returns. To simplify our analyses and presentations, we take into account the relation $\min \left\{X_{1}, \ldots \ldots, X_{n}\right\}=\max \left\{-X_{1}, \ldots,-X_{n}\right\}$ and multiply our returns series by -1 . 
Following Gettinby et al. (2006), we construct the $L$-moment ratio diagram for the annual maxima of all three of our data series (see Figure 3). These diagrams plot the estimated $L$-skewness against the corresponding estimated $L$ kurtosis. It is evidenced that these diagrams suggest that GEVD and GLD are closer to the annual maxima, indicating better fits, as compared to GPD. This is somewhat obvious as these maxima correspond to a BM approach with yearlysized blocks, whereas GPD, in contrast, arise from exceedances above a threshold. Further analyses will show that GLD and GEVD do not always outperform GPD in terms of risk modeling.

Figure 3. $L$-moment ratio diagram for annual minima of (a) US Mining Index, (b) SA Mining Index and (c) JSE Resource 10 Index

(a) US Mining Index

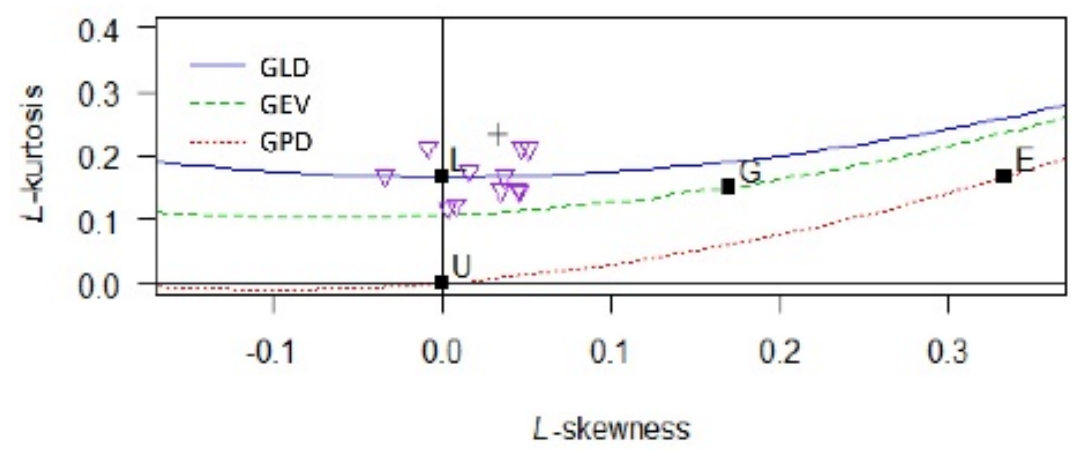

(b) SA Mining Index

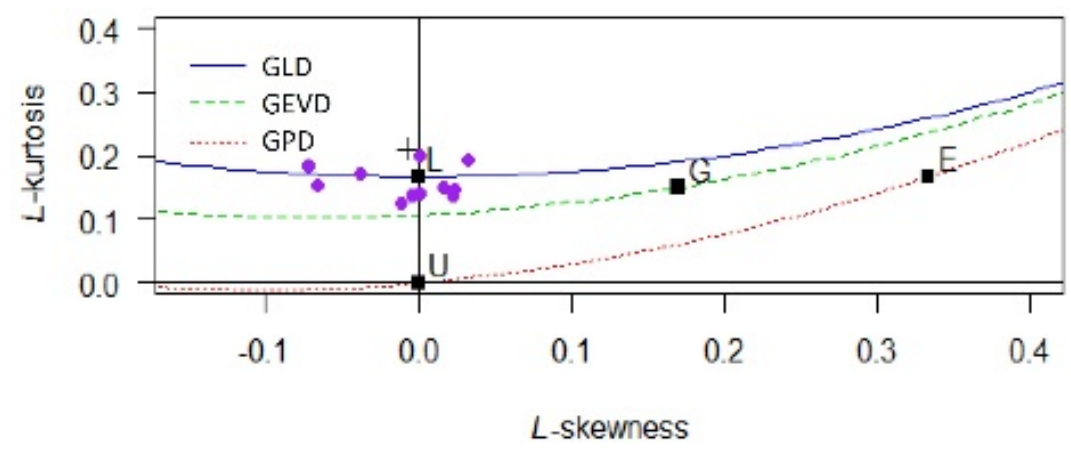

(c) JSE Resource 10 Index

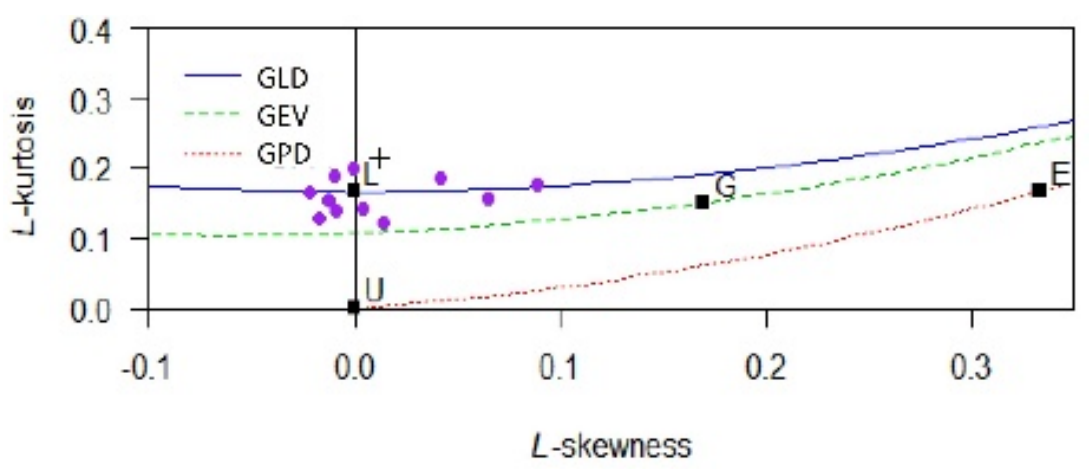


We fit GEVD and GLD to our return series using block sizes 5, 10 and 21 that arise naturally, corresponding to weekly minima, fortnightly minima and monthly minima, respectively. The residual plots and Q-Q plots for modeling Dow Jones US Mining Index negative returns with GEVD are presented in Figure 4.

Firstly, we observe that there are no recognizable patterns for the residual scatter plots, indicating independence of the estimation errors. Secondly, the Q-Q plots illustrate that, as the block size increases, slightly greater adequacy is depicted by the fitted GEVD model (with quantile points closer to the straight line). This is in accordance with the Fisher-Tippett-Gnedenko theorem. We have made similar observations for estimations with GLD, and for SA Mining Index and RESI.

To model a set of data with GPD, we first need to identify a suitable threshold value. A common method is to examine the mean excess plot and observe where the curve is approximately positively linear. Figure 5 presents the mean excess plot for each of our data sets and demonstrates that suitable threshold values are roughly between 0.01 and 0.05 . However, to choose a high enough threshold with a sufficient number of exceedances, we select the $85 \%, 90 \%$ and $95 \%$ quantiles, in each data series, as thresholds (all of which are between 0.01 and 0.05 ).

Figure 4. Residual plots (left) and Q-Q plots (right) for the fitting of GEVD to Dow Jones US Mining Index, with block sizes 5 (row 1), 10 (row 2) and 21 (row 3)
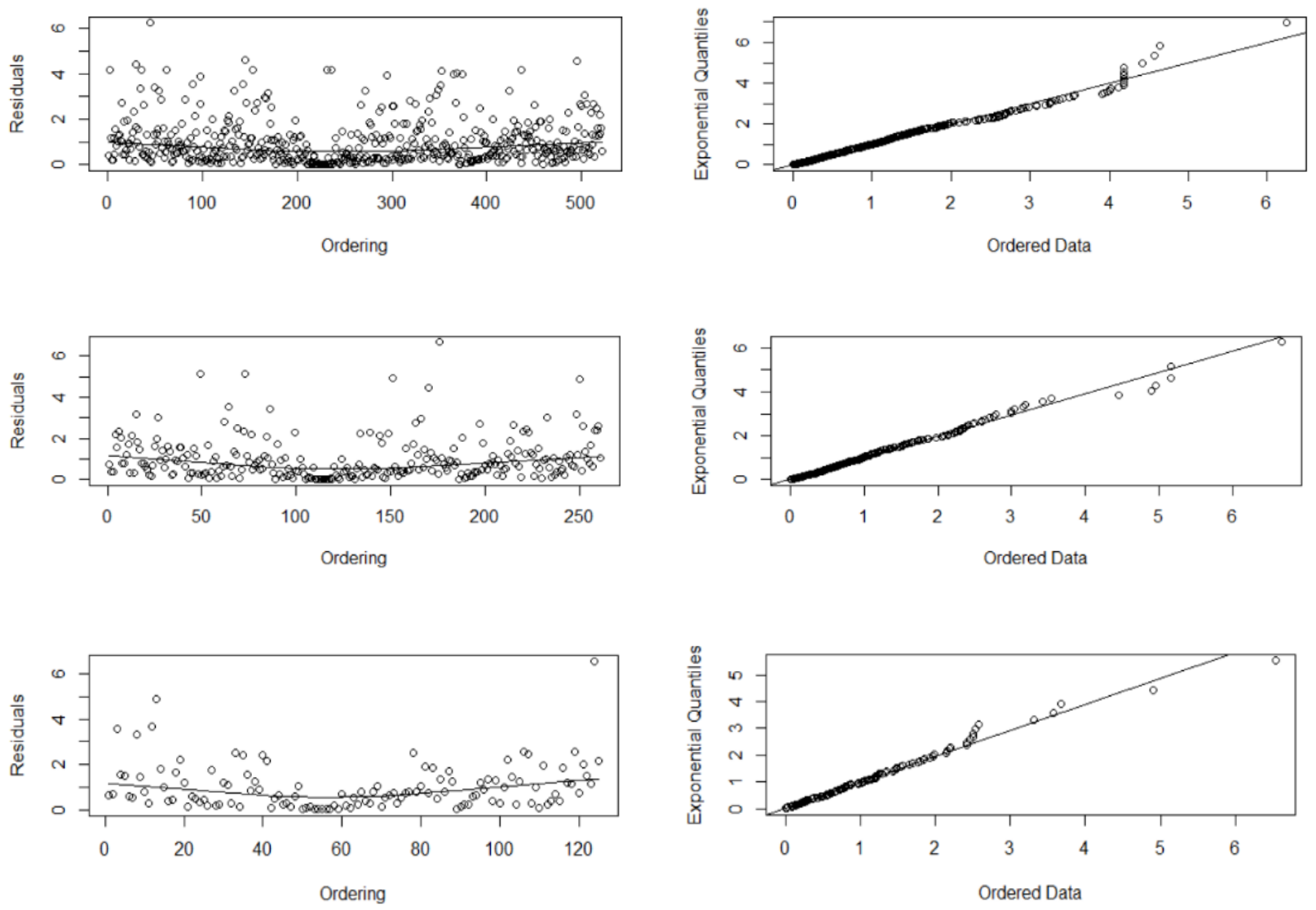
Figure 5. Mean excess plots for (a) Dow Jones US Mining Index, (b) SA Mining Index and (c) JSE Resource 10 Index

(a) Dow Jones US Mining Index

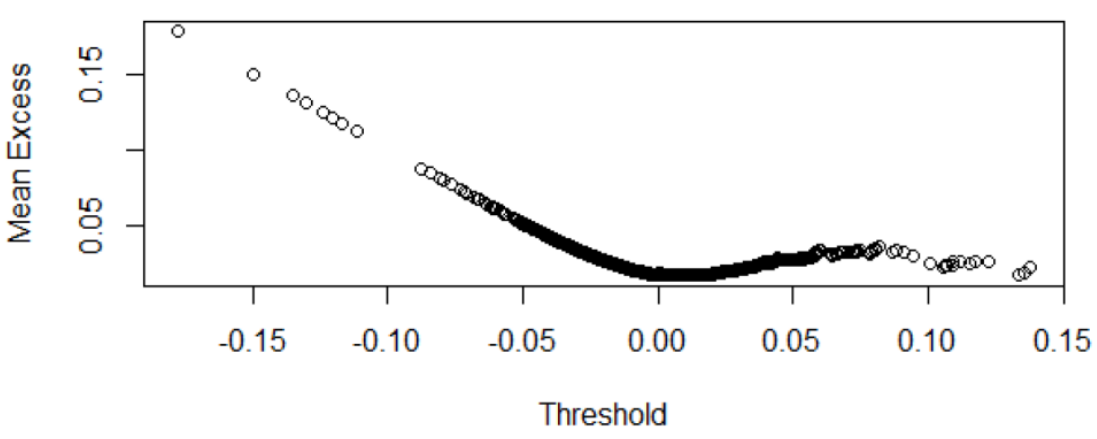

(b) SA Mining Index

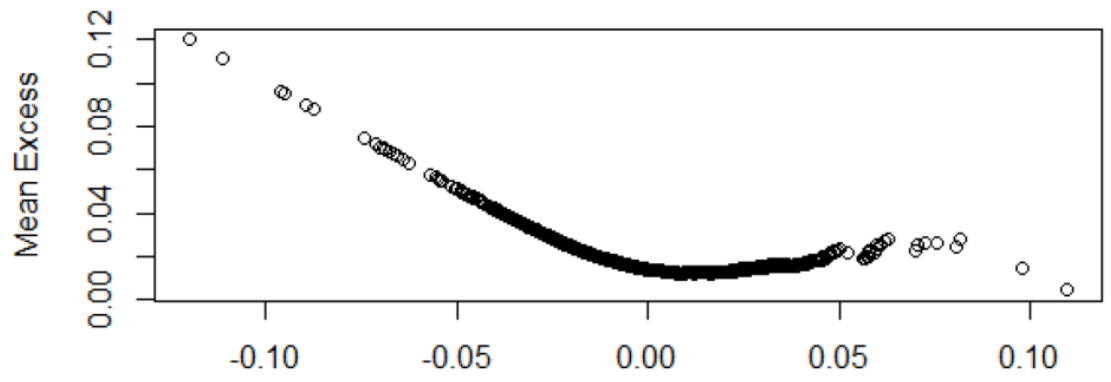

Threshold

(c) JSE Resource 10 Index

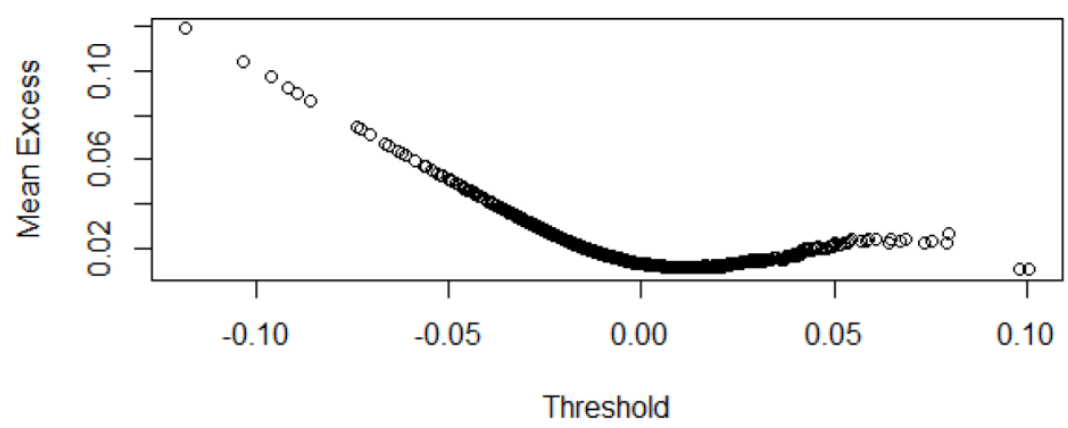

Figure 6 provides the residual plots and Q-Q plots for fitting GPD to the SA Mining Index, at threshold values of $85 \%$, $90 \%$ and $95 \%$ quantiles. Again, no distinct patterns are portrayed by the residual plots and the Q-Q plots indicate that GPD does provide suitable depiction of the exceedances. Although, very little difference is observed for the Q-Q plots at difference threshold levels. Similar observations were made for US Mining Index and RESI. 
Figure 6. Residual plots (left) and Q-Q plots (right) for the fitting of GPD to SA Mining Index, with threshold at 85\% (row 1), $90 \%$ (row 2) and $95 \%$ (row 3) quantiles
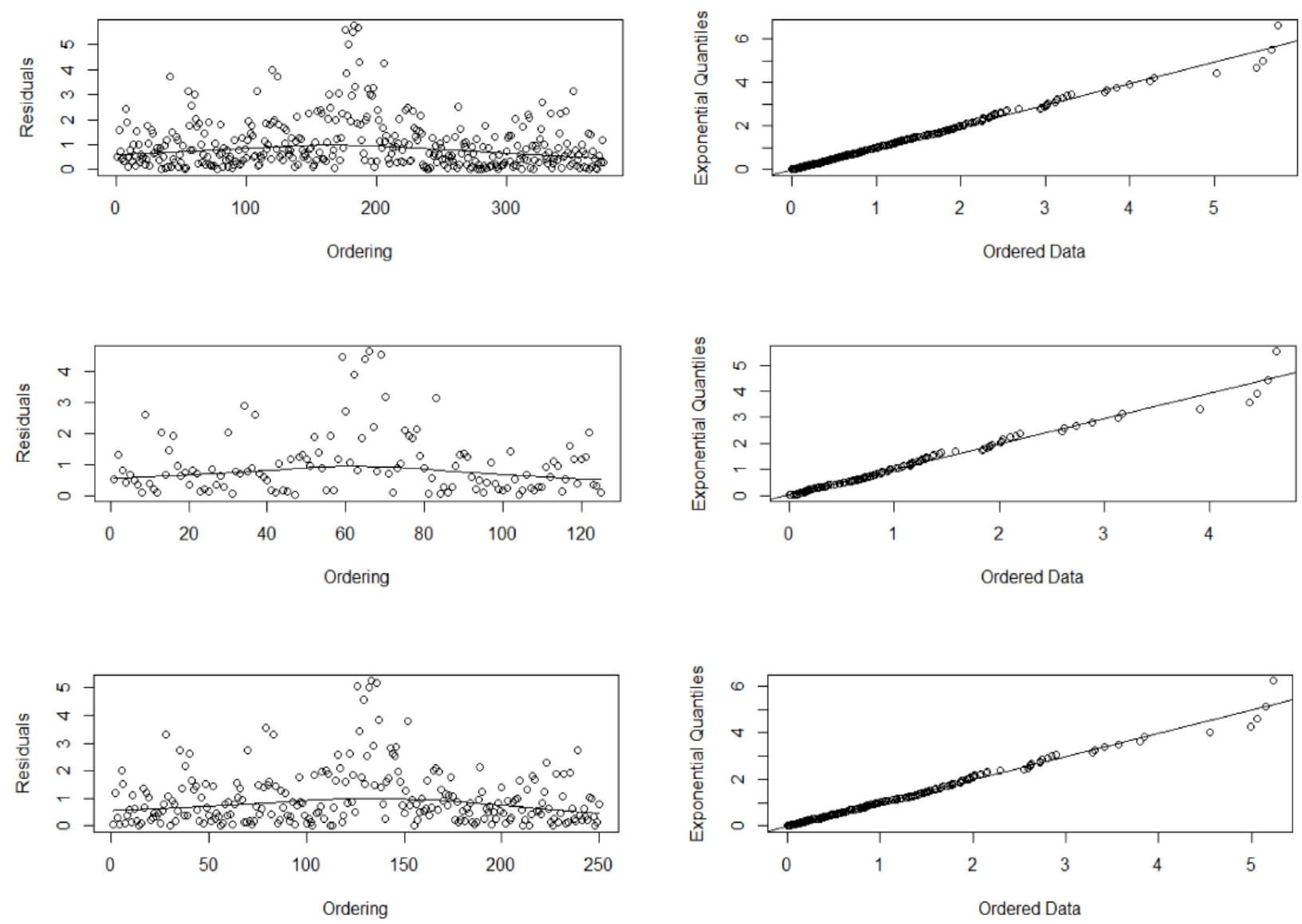

We now estimate VaR, at $0.1 \%, 1 \%$ and $5 \%$ levels, for the different indices using the EVT models, and calculate the number of $\mathrm{VaR}$ violations in each case. The results are provided in Table 2. Here, GEVD5 denotes the fitted GEVD model with block size 5, GLD5 denotes the fitted GLD model with block size 5, and henceforth. Whereas, GPD85, GPD90 and GPD95 denote the GPD models with threshold values at $85^{\text {th }}, 90^{\text {th }}$ and $95^{\text {th }}$ percentiles, respectively. The results are also contrasted with the classical Gaussian model.

It is clear from Table 2 that GLD resulted in the most number of violations at the $0.1 \%$ VaR level, for all three indices. However, this is less so when we move onto $1 \%$ and $5 \%$ levels. Evidently, the outcomes also show that the number of VaR violations digress more drastically with changing block sizes for GEVD and GLD, as compared to GPD, where only very minor changes are observed when the threshold level varies. As expected, the Gaussian model produced excess number of violations at high quantiles (i.e. at $0.1 \%$ and $1 \%$ levels) and insufficient number of violations at lower level quantiles (i.e. at $5 \%$ level). 
Table 2. Number of $\mathrm{VaR}$ backtesting violations at estimated $0.1 \%, 1 \%$ and $5 \%$ VaR levels

\begin{tabular}{l|c|c|c|c|c|c|c|c|c}
\hline \multirow{3}{*}{ Model } & \multicolumn{2}{|c|}{ Dow Jones US Mining Index } & \multicolumn{3}{c|}{ SA Mining Index } & \multicolumn{3}{|c}{ JSE Resource 10 Index } \\
& $\mathbf{0 . 1 \%}$ & $\mathbf{1 \%}$ & $\mathbf{5 \%}$ & $\mathbf{0 . 1 \%}$ & $\mathbf{1 \%}$ & $\mathbf{5 \%}$ & $\mathbf{0 . 1 \%}$ & $\mathbf{1 \%}$ & $\mathbf{5 \%}$ \\
& $\mathbf{( 2 )}$ & $\mathbf{( 2 6 )}$ & $\mathbf{( 1 3 0 )}$ & $\mathbf{( 2 )}$ & $\mathbf{( 2 5 )}$ & $\mathbf{( 1 2 5 )}$ & $\mathbf{( 2 )}$ & $\mathbf{( 2 5 )}$ & $\mathbf{( 1 2 5 )}$ \\
\hline Gaussian & 26 & 45 & 105 & 16 & 46 & 105 & 16 & 43 & 111 \\
\hline GEVD5 & 3 & 34 & 153 & 6 & 26 & 125 & 7 & 29 & 123 \\
\hline GEVD10 & 6 & 41 & 174 & 5 & 30 & 140 & 5 & 31 & 144 \\
\hline GEVD21 & 6 & 50 & 201 & 7 & 40 & 184 & 8 & 37 & 188 \\
\hline GLD5 & 17 & 44 & 142 & 8 & 31 & 125 & 9 & 33 & 125 \\
\hline GLD10 & 16 & 42 & 153 & 9 & 35 & 141 & 10 & 35 & 137 \\
\hline GLD21 & 16 & 45 & 179 & 8 & 40 & 181 & 10 & 37 & 182 \\
\hline GPD85 & 1 & 27 & 126 & 5 & 23 & 123 & 5 & 22 & 119 \\
\hline GPD90 & 1 & 27 & 125 & 4 & 23 & 124 & 4 & 24 & 125 \\
\hline GPD95 & 2 & 27 & 129 & 4 & 23 & 125 & 5 & 22 & 125 \\
\hline
\end{tabular}

Ideally, the proportion of violations should be equal to the corresponding VaR level. This hypothesis is tested using the Kupiec likelihood ratio test and the results are presented in Table 3. It is apparent that GPD is a suitable model for all three indices, while GEVD and GLD is rejected at several VaR levels. Inspecting each index, GPD models account for the highest $p$-value in most of the VaR levels. These are strong evidence that GPD is a more suitable model against GEVD and GLD, contrary to previous findings. We also notice that the most suitable choice of model varies across different VaR levels (highest $p$-value varies across VaR levels for each index). This agrees with findings suggested by Huang et al. (2014).

Table 3. VaR backtesting using the Kupiec likelihood ratio test

\begin{tabular}{l|c|c|c|c|c|c|c|c|c}
\hline \multirow{2}{*}{ Model } & \multicolumn{3}{|c|}{ Dow Jones US Mining Index } & \multicolumn{3}{c|}{ SA Mining Index } & \multicolumn{3}{c}{ JSE Resource 10 Index } \\
\cline { 2 - 11 } & $\mathbf{0 . 1 \%}$ & $\mathbf{1 \%}$ & $\mathbf{5 \%}$ & $\mathbf{0 . 1 \%}$ & $\mathbf{1 \%}$ & $\mathbf{5 \%}$ & $\mathbf{0 . 1 \%}$ & $\mathbf{1 \%}$ & $\mathbf{5 \%}$ \\
\hline Gaussian & $<0.0001$ & 0.0007 & 0.0183 & $<0.0001$ & 0.0002 & 0.0594 & $<0.0001$ & 0.0010 & 0.1907 \\
\hline GEVD5 & $\mathbf{0 . 8 1 2 6}$ & 0.1365 & 0.0479 & 0.0610 & $\mathbf{0 . 8 4 1 7}$ & $\mathbf{1}$ & 0.0199 & 0.4330 & 0.8540 \\
\hline GEVD10 & 0.0728 & 0.0067 & 0.0002 & 0.1643 & 0.3299 & 0.0323 & 0.1643 & 0.2450 & 0.0883 \\
\hline GEVD21 & 0.0728 & $<0.0001$ & $<0.0001$ & 0.0199 & 0.0055 & $<0.0001$ & 0.0058 & 0.0243 & $<0.0001$ \\
\hline GLD5 & $<0.0001$ & 0.0013 & 0.3039 & 0.0058 & 0.2450 & $\mathbf{1}$ & 0.0015 & 0.1253 & $\mathbf{1}$ \\
\hline GLD10 & $<0.0001$ & 0.0040 & 0.0479 & 0.0015 & 0.0580 & 0.1498 & 0.0004 & 0.0580 & 0.2779 \\
\hline GLD21 & $<0.0001$ & 0.0007 & $<0.0001$ & 0.0058 & 0.0055 & $<0.0001$ & 0.0004 & 0.0243 & $<0.0001$ \\
\hline GPD85 & 0.2542 & $\mathbf{0 . 8 5 7 1}$ & 0.6910 & 0.1643 & 0.6836 & 0.8540 & 0.1643 & 0.5382 & 0.5790 \\
\hline GPD90 & 0.2542 & $\mathbf{0 . 8 5 7 1}$ & 0.6253 & $\mathbf{0 . 3 8 3 0}$ & 0.6836 & 0.9268 & $\mathbf{0 . 3 8 3 0}$ & $\mathbf{0 . 8 3 9 6}$ & $\mathbf{1}$ \\
\hline GPD95 & 0.6944 & $\mathbf{0 . 8 5 7 1}$ & $\mathbf{0 . 8 9 9 7}$ & $\mathbf{0 . 3 8 3 0}$ & 0.6836 & $\mathbf{1}$ & 0.1643 & 0.5382 & $\mathbf{1}$ \\
\hline
\end{tabular}

We also consider each index individually. For US Mining Index, only estimates from GPD models are not rejected at all three VaR levels, at 5\% level of test significance. All three VaR levels of SA Mining Index are adequately depicted by GEVD5 and the three GPD models. Lastly, GEVD10 and GPD estimates are not rejected for RESI, at 5\% level of test significance. It is also interesting to note that GLD does not in general produce suitable VaR estimates across all levels. However, it does produce high $p$-values at several VaR levels. In particular, it outperforms the corresponding GEVD estimates at 5\% VaR level. 
Table 4. ES backtesting using the bootstrap $t$-test

\begin{tabular}{l|c|c|c|c|c|c|c|c|c}
\hline \multirow{2}{*}{ Model } & \multicolumn{2}{|c|}{ Dow Jones US Mining Index } & \multicolumn{3}{c|}{ SA Mining Index } & \multicolumn{3}{c}{ JSE Resource 10 Index } \\
\cline { 2 - 11 } & $\mathbf{0 . 1 \%}$ & $\mathbf{1 \%}$ & $\mathbf{5 \%}$ & $\mathbf{0 . 1 \%}$ & $\mathbf{1 \%}$ & $\mathbf{5 \%}$ & $\mathbf{0 . 1 \%}$ & $\mathbf{1 \%}$ & $\mathbf{5 \%}$ \\
\hline Gaussian & $<0.0001$ & $<0.0001$ & $<0.0001$ & 0.0014 & 0.0026 & $<0.0001$ & 0.0024 & 0.0003 & $<0.0001$ \\
\hline GEVD5 & 0.5490 & 0.5104 & 0.5202 & 0.4474 & 0.5235 & 0.5235 & 0.4841 & 0.5134 & 0.5090 \\
\hline GEVD10 & 0.5209 & 0.4918 & 0.5224 & 0.4320 & 0.5211 & 0.5212 & 0.4861 & 0.5076 & 0.5192 \\
\hline GEVD21 & 0.5235 & 0.4949 & 0.5300 & 0.4662 & 0.5269 & 0.5187 & 0.4982 & 0.5198 & 0.5116 \\
\hline GLD5 & 0.5057 & 0.5011 & 0.5005 & 0.4909 & 0.5247 & 0.5254 & 0.5094 & 0.5292 & 0.5141 \\
\hline GLD10 & 0.5002 & 0.5113 & 0.5068 & 0.4866 & 0.5277 & 0.5103 & 0.4952 & 0.5009 & 0.5250 \\
\hline GLD21 & 0.5132 & 0.4991 & 0.4950 & 0.4951 & 0.5371 & 0.5141 & 0.4924 & 0.5310 & 0.5114 \\
\hline GPD85 & 0.5145 & 0.5079 & 0.5194 & 0.4470 & 0.5241 & 0.5243 & 0.4893 & 0.5146 & 0.5139 \\
\hline GPD90 & 0.5201 & 0.5254 & 0.5226 & 0.4976 & 0.5305 & 0.5131 & 0.4588 & 0.5277 & 0.5185 \\
\hline GPD95 & 0.5105 & 0.5191 & 0.5205 & 0.5061 & 0.5201 & 0.5111 & 0.4992 & 0.5045 & 0.5160 \\
\hline
\end{tabular}

Table 4 displays the $p$-values from backtests of ES, using the bootstrap $t$-test, for the indices. It is clear that very little differences exists between our fitted EVT models. However, all EVT models have produced suitable ES estimates for each index, at all VaR levels, and clearly outperform the Gaussian model (rejected at all levels for all indices).

As an attempt to reduce the look-back bias, we also implement out-of-sample tests for each model on the three indices. The data range used for out-of-sample testing is 25 June 2014 to 4 May 205 (224 daily returns), for US Mining Index, and 10 September 2014 to 4 May 2015 (169 daily returns), for SA Mining index and RESI. However, we are only able to obtain results for $5 \% \mathrm{VaR}$ levels due to limited data. The results of these tests are presented in Tables 5, 6 and 7.

Table 5. Number of VaR out-of-sample violations at estimated 5\% VaR levels

\begin{tabular}{l|c|c|c}
\hline \multirow{2}{*}{ Model } & Dow Jones US Mining Index & SA Mining Index & JSE Resource 10 Index \\
\cline { 2 - 4 } & $\mathbf{( 1 1 )}$ & $\mathbf{( 8 )}$ & $\mathbf{( 8 )}$ \\
\hline Gaussian & 3 & 9 & 8 \\
\hline GEVD5 & 5 & 10 & 11 \\
\hline GEVD10 & 7 & 12 & 15 \\
\hline GEVD21 & 8 & 12 & 9 \\
\hline GLD5 & 5 & 10 & 11 \\
\hline GLD10 & 5 & 11 & 14 \\
\hline GLD21 & 7 & 12 & 9 \\
\hline GPD85 & 3 & 10 & 9 \\
\hline GPD90 & 3 & 10 & 9 \\
\hline GPD95 & 4 & 11 & \\
\hline
\end{tabular}

Table 6. Out-of-sample Kupiec likelihood ratio test for 5\% VaR

\begin{tabular}{l|c|c|c}
\hline \multicolumn{1}{c}{ Model } & Dow Jones US Mining Index & SA Mining Index & JSE Resource 10 Index \\
\hline Gaussian & 0.0030 & 0.8476 & 0.5984 \\
\hline GEVD5 & 0.0336 & 0.5945 & 0.8727 \\
\hline GEVD10 & 0.1678 & 0.2373 & 0.3888 \\
\hline GEVD21 & 0.3022 & 0.2373 & 0.0362 \\
\hline GLD5 & 0.0336 & 0.5945 & 0.8476 \\
\hline GLD10 & 0.0336 & 0.3888 & 0.3888 \\
\hline GLD21 & 0.1678 & 0.2373 & 0.0723 \\
\hline GPD85 & 0.0030 & 0.5945 & 0.8727 \\
\hline GPD90 & 0.0030 & 0.5945 & 0.8476 \\
\hline GPD95 & 0.0114 & 0.3888 & 0.8476 \\
\hline
\end{tabular}

Firstly, the Gaussian model has again provided unsuitable VaR estimates for US Mining Index returns. More interestingly though, it resulted in more adequate VaR estimates for returns in SA Mining Index and RESI. Furthermore, interesting comparisons can be drawn from comparing the three EVT models for different data. For US 
Mining Index, it can be observed that GEVD and GLD performs slightly better than GPD, as suggested by some prior research such as Gettinby et al. (2006) and Tolikas and Gettinby (2009). However, the two groups become more comparable for SA Mining Index and GPD is certainly the better overall performer for RESI. The results may be attributed to the significantly higher kurtosis and volatility in the US Mining returns.

Table 7. Out-of-sample bootstrap $t$-test for $5 \% \mathrm{ES}$

\begin{tabular}{l|c|c|c}
\hline \multicolumn{1}{c|}{ Model } & Dow Jones US Mining Index & SA Mining Index & JSE Resource 10 Index \\
\hline Gaussian & 0.0312 & 0.5663 & 0.2627 \\
\hline GEVD5 & 0.8685 & 0.8670 & 0.6324 \\
\hline GEVD10 & 0.9298 & 0.8639 & 0.7297 \\
\hline GEVD21 & 0.9108 & 0.7164 & 0.7720 \\
\hline GLD5 & 0.9206 & 0.8729 & 0.7048 \\
\hline GLD10 & 0.8665 & 0.8588 & 0.7668 \\
\hline GLD21 & 0.9230 & 0.7188 & 0.7180 \\
\hline GPD85 & 0.8561 & 0.8763 & 0.6513 \\
\hline GPD90 & 0.8756 & 0.8891 & 0.7108 \\
\hline GPD95 & 0.9166 & 0.9131 & 0.7034 \\
\hline
\end{tabular}

The comparative adequacy across different EVT models for ES is less distinguishable, as for the in-sample ES testing. However, their superiority over the Gaussian model is more emphasized by the bootstrap $t$-test, where magnitudes of exceedances are taken into account.

\section{CONCLUSION}

In this article, we have examined the suitability of EVT models, namely GEVD, GLD and GPD, in estimating VaR and ES for US Mining Index, SA Mining Index and RESI. GEVD and GLD were fitted using the BM approach and GPD was fitted by the POT method. Adequacy of the resulting VaR and ES estimates were tested using the Kupiec likelihood ratio test and the bootstrap $t$-test, respectively.

Contrary to previous findings, our results have shown that GLD does not always outperform GEVD and GPD, in terms of VaR and ES assessments. In particular, our in-sample tests showed GPD as a better model in general, across all VaR levels. This is evidenced from observing that GPD is not rejected by the Kupiec likelihood ratio test at all $\mathrm{VaR}$ levels. However, the out-of-sample tests for US Mining Index indicated GPD performing worse than GEVD and GLD, while almost the opposite can be said for SA Mining Index and RESI. We suggest such difference is attributed to the significantly higher kurtosis and volatility in the US Mining returns and signifies that the adequacy of risk models may differ between data from a developed market or an emerging market. Lesser difference between the EVT models is observed for ES backtesting, for all three indices. However, the bootstrap $t$-tests can further emphasize the superiority of EVT models over the classical Gaussian model.

Suggestions for further work may include comparing GEVD, GLD and GPD in the context of VaR and ES estimation using GARCH-based approaches. We did not incorporate our EVT models into a GARCH model because we wanted to compare our results directly with previous work involving GLD. However, it would be standard procedure to fit a GARCH model. Comparison of EVT models across other financial sectors, and their contrasts to the resource sector, may also be of interest. On the other hand, one may analyze whether there may be significant difference in model performance depending on the time period, e.g., pre-crisis versus post-crisis.

\section{AUTHOR BIOGRAPHIES}

Chun-Kai Huang is a lecturer in Statistics at the University of Cape Town and an honorary lecturer at the University of KwaZulu-Natal. He is a member of the South African Statistical Association and a fellow of the Cambridge Commonwealth Society. His research interests lie in probabilistic exchangeability, financial risk modeling, moment problems, extreme value analysis and multiple-phase sampling with auxiliary variables. Email: chunkai.huang@ukzn.ac.za (corresponding author) 
Venelle Pather is a financial modeler at J Maynard. She completed her BScHons in Statistics in 2014, with distinction, at the University of KwaZulu-Natal. Her research interests lie in financial modeling and extreme value analysis. Email: venelle.pather@gmail.com

Jahvaid Hammujuddy is a lecturer in Statistics at the University of KwaZulu-Natal. He is a member of the South African Statistical Association and the Institute of Certificated and Chartered Statisticians of South Africa. His research interests lie in multivariate analysis, generalized linear mixed models and extreme value analysis. Email: hammujuddy@ukzn.ac.za

Knowledge Chinhamu is a lecturer in Statistics at the University of KwaZulu-Natal and a member of the South African Statistical Association. His research interests lie in financial time series and econometric modeling. Email: chinhamu@ukzn.ac.za

\section{REFERENCES}

Acerbi, C., \& Tasche, D. (2002). Expected Shortfall: a natural coherent alternative to Value at Risk. Economic Notes, 31(2), $379-388$. Anđelić, G., Milošev, I., \& Đaković, V. (2010). Extreme value theory in emerging markets. Economic Annals, 55(185), 63-105. Bali, T. G. (2003). An extreme value approach to estimating volatility and value at risk. The Journal of Business, 76(1), 83-108. Balkema, A., \& de Haan, L. (1974). Residual life time at great age. Annals of Probability, 2, 792-804.

Basel (2012). Basel Committee on Banking Supervision - Fundamental review of the trading book. Bank for International Settlements. Retrieved from http://www.bis.org/publ/bcbs219.pdf

Brodin, E., \& Kluppelberg, C. (2008). Extreme value theory in finance, in Encyclopedia of Quantitative Risk Analysis and Assessment. Wiley, Chichester.

Chinhamu, K., Huang, C-K., Huang, C-S., \& Hammujuddy, J. (2014). Empirical analyses of extreme value models for the South African mining index. South African Journal of Economics, 83(1), 41-55.

Coles, S. (2001). An Introduction to Statistical Modelling of Extreme Values. Springer-Verlag, London.

Danielsson, J., \& de Vries, C. G. (2000). Value-at-risk and extreme returns. Annales d'Économie et de Statistique, (60), $239-270$.

Embrechts, P., Klüppelberg, C., \& Mikosh, T. (1997). Modelling Extremal Events: For Insurance and Finance. Berlin: Springer-Verlag.

Embrechts, P., Resnick, S., \& Samorodnitsky, G. (1999). Extreme value theory as a risk management tool. North American Actuarial Journal, 3(2), 30-41.

Fernandez, V. (2003). Extreme value theory and value at risk. Revista de Análisis Económico, 18(1), 57-85.

Fisher, R. A., \& Tippett, L. H. C. (1928). Limiting forms of the frequency distribution of the largest and smallest member of a sample. Mathematical Proceedings of the Cambridge Philosophical Society, 24(2), 180-190.

Gençay, R. \& Selçuk, F. (2004). Extreme value theory and value-at-risk: Relative performance in emerging markets. International Journal of Forecasting, 20, 287-303.

Gettinby, G. D., Sinclair, C. D., \& Brown, R. A. (2006). An analysis of the distribution of extremes in indices of share returns in the US, UK and Japan from 1963 to 2000. International Journal of Finance and Economics, 11, 97-113.

Gnedenko, B. V. (1943). Sur la distribution limite du terme maximum d'une serie aleatoire. Annals of Mathematics, (44), 423-453.

Huang, C-S., Huang, C-K., \& Chinhamu, K. (2014). Assessing the relative performance of heavy-tailed distributions: Empirical evidence from the Johannesburg Stock Exchange. The Journal of Applied Business Research, 30(4), 1263-1286.

Koedijk, K. G., Schafgans, M. M. A., \& de Vries, C. G. (1990). The tail index of exchange rate returns. Journal of International Economics, 29(1-2), 93-108.

Kupiec, P. H. (1995). Techniques for verifying the accuracy of risk management models. Journal of Derivatives, 3(2), 73-84.

Levine, D. (2009). Modeling tail behavior with extreme value theory. Risk Management, 17, 14-18.

McNeil, A. J., \& Frey, R. (2000). Estimation of tail-related risk measures for heteroscedastic financial time series: an extreme value approach. Journal of Empirical Finance, 7(3-4), 271-300.

Nidhin, K., \& Chandran, C. (2013). Importance of generalized logistic distribution in extreme value modeling. Applied Mathematics, 4 , 560-573.

Pickands, J. (1975). Statistical inference using extreme order statistics. Annals of Statistics, 3, 119-131.

Shapiro, D. (2012). Long-term extremes of South African financial and economic variables. Proceedings of the Actuarial Society of South Africa's 2012 Convention, 167-194.

Seymour, A. J., \& Polakow, D. A. (2003). A coupling of extreme-value theory and volatility updating with value-at-risk estimation in emerging markets: a South African test. Multinational Finance Journal, 7(1-2), 3-23.

Tolikas, K., \& Brown, R. A. (2006). The distribution of the extreme daily share returns in the Athens stock exchange. The European Journal of Finance, 12(1), 1-22.

Tolikas, K., \& Gettinby, G. D. (2009). Modelling the distribution of the extreme share returns in Singapore. Journal of Empirical Finance, 16, 179-186.

Tolikas, K., Koulakiotis, A., \& Brown, R. A. (2007). Extreme risk and value-at-risk in the German stock market. The European Journal of Finance, 13(4), 373-395. 